\title{
COMMENT
}

Check for updates

\section{Platform trials and the future of evaluating therapeutic behavioural interventions}

${ }^{1}$ Charité - Universitätsmedizin Berlin, Klinik für Psychiatrie und Psychotherapie, Campus Benjamin Franklin, Berlin, Germany.

${ }^{2}$ Charité - Universitätsmedizin Berlin, Med. Klinik m.S. Psychosomatik, Campus Benjamin Franklin, Berlin, Germany.

${ }^{3}$ Institut für Neuroimmunologie und MS (INIMS), Zentrum für Molekulare Neurobiologie,

Universitätsklinikum

Hamburg-Eppendorf,

Hamburg, Germany.

${ }^{4}$ Center for Medical Statistics, Informatics, and Intelligent Systems, Medical University of Vienna, Vienna, Austria.

${ }^{5}$ CRONICAS Centre of Excellence in Chronic Diseases, Universidad Peruana Cayetano Heredia, Lima, Peru.

${ }^{6}$ Department of Medicine, School of Medicine Universidad Peruana Cayetano Heredia, Lima, Peru.

${ }^{7}$ The George Institute for Global Health, University of New South Wales, Sydney, New South Wales, Australia.

${ }^{8}$ Faculty of Epidemiology and Population Health, London School of Hygiene and Tropical Medicine, London, UK

${ }^{9}$ Department of Psychological Medicine, Institute of Psychiatry, Psychology and Neuroscience, King's College London, London, UK.

凶e-mail: stefan.gold@ charite.de

\section{Stefan M. Gold $\mathbb{1}^{1,2,3 凶}$, Marta Bofill Roig $^{4}$, J. Jaime Miranda $\mathbb{(}^{5,6,7,8}$, Carmine Pariante ${ }^{9}$, Martin Posch $\mathbb{D}^{4}$ and Christian Otte (iD) ${ }^{1}$}

Therapeutic interventions are typically evaluated in individual, parallel group trials, which are time consuming and provide limited information on comparative efficacy. Clinical psychology should leverage advances in other fields to improve and accelerate the evaluation process by adopting more efficient platform trials.

Many disciplines in psychology, including clinical psychology, aim to improve well-being by deploying behavioural interventions such as psychotherapy, educational programmes and dietary interventions. Clinical trials, where the effect of an intervention of interest is compared to a control condition, are used to evaluate whether interventions are safe and effective.

These trials are typically set up to answer a specific question about one intervention and are registered in trial registries accordingly, with specific start and end dates. Consequently, the infrastructure for recruitment, intervention delivery and outcome assessment is usually set up for every trial and dismantled at its end. Although this is effective in the sense that a 'one intervention, one trial' approach will eventually determine whether an intervention works and is safe, the process is inefficient.

Furthermore, there is no regulatory oversight for behavioural interventions, so binding guidance on design factors such as the measures used, timing of assessments, intervention duration and intensity, participant inclusion and exclusion criteria, and control condition used, is minimal. As a result, comparability across trials is hampered.

By contrast, platform trials use a shared infrastructure, in which several interventions are deployed under the same 'master protocol' and tested against a shared control condition (FIG. 1). Platform trials have known potential for drug development ${ }^{1}$. The COVID-19 pandemic accelerated the development and implementation of platform trials ${ }^{2}$. For example, the RECOVERY platform trial quickly established an effective treatment for patients hospitalized with SARS-CoV-2 (the use of dexamethasone) that immediately changed clinical practice $^{3}$. Although platform trials are becoming more accepted, this discussion has been largely limited to the drug development space. However, the recent successes of platform trials offer opportunities to leverage these advances for other areas, such as psychology.

\section{Benefits}

Compared with traditional trials, platform trials improve the comparability of observed treatment effects. First, the shared control arm minimizes heterogeneity in comparator groups. This is important in psychology, in which true placebo conditions do not exist and treatment as usual, a common comparator, can have a wide range of meanings in different settings ${ }^{4}$. Second, platform trials generate comparative evidence from several active arms rather than relying on indirect comparisons of effect sizes obtained from individual trials with different control groups.

Platform trials are also more efficient than traditional trials in terms of the required sample size (FIG. 1), and therefore the time needed for full recruitment. Furthermore, the shared control condition enables a larger proportion of participants to be assigned to experimental arms than in traditional trials. This is particularly relevant for trials of behavioural interventions, in which wait-list control groups that receive no treatment are frequently used ${ }^{5,6}$.

To further increase efficiency in platform trials, preset interim analyses can be used ${ }^{7}$ to determine whether treatment arms should be terminated early (for example, if an interim analysis suggests an effect with a low probability of success if tested in a subsequent confirmatory trial). Similarly, more participants can be assigned to more promising experimental arms while poorly performing experimental arms receive fewer participants. These measures can also be considered more ethical: they reduce the number of patients assigned to treatment arms that turn out to be ineffective.

\section{Challenges}

Platform trials typically run over a longer time period than an individual trial. This can lead to chronological bias, resulting in time trends in the outcome measure. These trends might be caused by changes in the standard 
a Individual randomized controlled trials Overall sample size: $N=1,000$

Trial 1

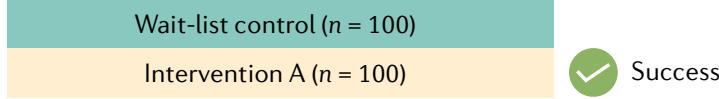

Trial 2

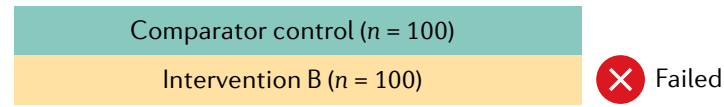

Trial 3

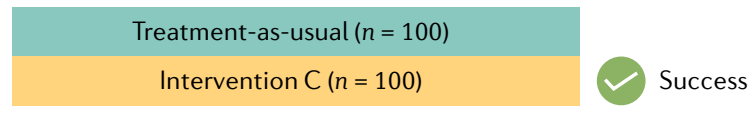

Trial 4

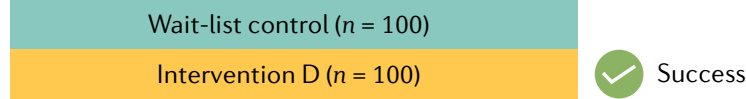

Trial 5

Wait-list control $(n=100)$

Intervention $\mathrm{E}(n=100)$

Time

b Platform trial

Overall sample size: approx. $N=550$

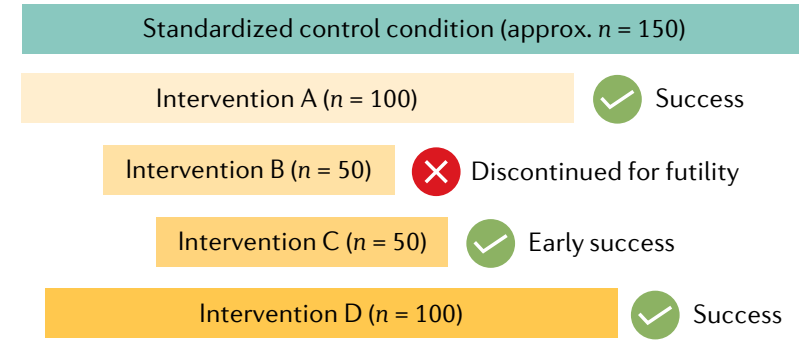

Intervention $\mathrm{E}(n=100)$

Failed

Time

Fig. 1 | Comparison of traditional clinical trials and a platform trial for five

behavioural interventions. a | In a traditional approach, independently run randomized controlled trials each test one experimental intervention. $\mathbf{b}$ | In a platform trial, a shared infrastructure is used to test several treatments against a shared control condition. New treatment arms can be added or discontinued over time. This approach uses resources more efficiently because the overall sample size, and the number of participants in control conditions, are lower (assuming equal effect sizes in both scenarios).

of care, adding or dropping of participating centres, shifts in the patient population, systematic variations in participants' responses to the control condition or changes in expectancy effects when a new treatment that is perceived as particularly promising enters or leaves the trial. Such time trends have to be adjusted for in the analysis.

Platform trials also rely on the assumption that all interventions are equally acceptable to patients, which might not be the case. If certain treatments are more (or less) acceptable to participants, this might influence their decision to enrol or lead to systematic differences in attrition between trial arms, undermining comparability.

Researchers must conduct comprehensive pre-trial simulations to estimate how the platform trial will perform under different scenarios. This is necessary because platform trials have many adaptive features and it is important to model their implications for trial feasibility.
However, considerable experience is needed to run these simulations. Thus, platform trials require specialized statistical expertise.

Finally, platform trials require a shared infrastructure that must be established, maintained and governed by some entity. Government ministries or agencies, health insurers or charities might be interested in serving in this capacity, but might not have the means or experience necessary. Moreover, platform trial design is often a compromise and not 'tailored' to one specific intervention. Intervention 'owners' may therefore be reluctant to join such a trial, as it would mean relinquishing control of the experimental parameters to this entity.

\section{Outlook}

Platform trials might be particularly well suited to digital mental health interventions (DMHIs) ${ }^{8}$. As they are more resource efficient, platform trials for DMHIs could be implemented in settings with limited resources, such as low-income and middle-income countries ${ }^{9}$. This could have a substantial effect on improving mental health care globally. Moreover, the speed of platform trials might help to overcome the intrinsic problems of keeping up with the rapid pace of DMHIs, where the features being tested have to be frequently updated because of technological progress ${ }^{10}$.

Platform trials could have a transformative effect on clinical psychology more broadly by increasing the speed with which treatment effects are estimated, getting effective interventions into clinical care more quickly, and providing much needed comparisons between different interventions for the same condition. This would ultimately enable patients, care providers and payers to make better informed decisions and achieve better outcomes.

1. Adaptive Platform Trials Coalition. Adaptive platform trials: definition, design conduct and reporting considerations. Nat. Rev. Drug Discov. 18, 797-807 (2019).

2. Collignon, O., Burman, C. F., Posch, M. \& Schiel, A. Collaborative platform trials to fight COVID-19: methodological and regulatory considerations for a better societal outcome. Clin. Pharmacol. Ther. 110, 311-320 (2021).

3. RECOVERY Collaborative Group. Dexamethasone in hospitalized patients with Covid-19. N. Engl. J. Med. 384, 693-704 (2021).

4. Freedland, K. E., Mohr, D. C., Davidson, K. W. \& Schwartz, J. E.

Usual and unusual care: existing practice control groups

in randomized controlled trials of behavioral interventions.

Psychosom. Med. 73, 323-335 (2011).

5. Gold, S. M. et al. Control conditions for randomised trials of behavioural interventions in psychiatry: a decision framework. Lancet Psychiatry 4, 725-732 (2017).

6. Furukawa, T. A et al. Waiting list may be a nocebo condition in psychotherapy trials: a contribution from network meta-analysis. Acta Psychiatr. Scand. 130, 181-192 (2014).

7. Meyer, E. L. et al. The evolution of master protocol clinical trial designs: a systematic literature review. Clin. Ther. 42, 1330-1360 (2020).

8. Karyotaki, E. et al. Internet-based cognitive behavioral therapy for depression: a systematic review and individual patient data network meta-analysis. JAMA Psychiat. 78, 361-371 (2021).

9. Araya, R. et al. Effect of a digital intervention on depressive symptoms in patients with comorbid hypertension or diabetes in Brazil and Peru: two randomized clinical trials. J. Am. Med. Assoc 325, 1852-1862 (2021)

10. Mohr, D. C. et al. Trials of intervention principles: evaluation methods for evolving behavioral intervention technologies. J. Med. Internet Res. 17, e 166 (2015).

\section{Competing interests}

S.M.G., M.B.R., C.P., M.P., and C.O. are members of the public-private initiative EU-PEARL, funded by the European Commission, which develops reusable integrative research platforms for drug development in a range of diseases (including depression). J.J.M. declares no competing interests. 